\title{
Short communication: In vitro antimicrobial susceptibility of Mycoplasma bovis isolates identified in milk from dairy cattle in Belgium, Germany, and Italy
}

\author{
A. Barberio, ${ }^{*} \dagger^{1}$ B. Flaminio, ${ }^{*}$ S. De Vliegher,ł K. Supré,§ V. Kromker,\# C. Garbarino,॥l N. Arrigoni,॥ \\ G. Zanardi,II L. Bertocchi,II F. Gobbo, ${ }^{\star}$ I S. Catania, ${ }^{*}$ and P. Moroni $\dagger^{\star *}$ \\ *Istituto Zooprofilattico Sperimentale delle Venezie, viale dell'Università 10, 3020 Legnaro (PD), Italy \\ †Dipartimento di Medicina Veterinaria, Università degli Studi di Milano, Via Celoria 10, 20133, Milan, Italy \\ $\ddagger$ M-team and Mastitis and Milk Quality Research Unit, Department of Reproduction, Obstetrics and Herd Health, Faculty of Veterinary Medicine, \\ Ghent University, B-9820 Merelbeke, Belgium \\ §Flanders Milk Control Center, Hagenbroeksesteenweg 167, 2500 Lier, Belgium \\ \#University of Applied Sciences and Arts, Department of Bioprocess Engineering-Microbiology, Heisterbergallee 12, D-30453 Hannover, Germany \\ IIIstituto Zooprofilattico Sperimentale della Lombardia e dell'Emilia Romagna, Via Bianchi 9, 25124 Brescia, Italy \\ TDipartimento di Medicina Animale, Produzioni e Salute (MAPS), Università degli Studi di Padova, Viale dell'Università 16, 35020 Legnaro, \\ (PD), Italy \\ ${ }^{* *}$ Quality Milk Production Services, Animal Health Diagnostic Center, Cornell University, 240 Farrier Road, Ithaca, NY 14853
}

\section{ABSTRACT}

The objective of this study was to assess the in vitro antimicrobial susceptibility of 73 isolates of $\mathrm{Myco-}$ plasma bovis isolated from milk of dairy cattle herds of Belgium, Germany, and Italy. Minimal inhibitory concentration (MIC) values were determined by the microbroth dilution method for the following antimicrobials: erythromycin, spiramycin, tilmicosin, tylosin, lincomycin, enrofloxacin, doxycycline, oxytetracycline, florfenicol, and tiamulin. Macrolides, florfenicol, oxytetracycline, and enrofloxacin, were chosen because they represent antimicrobials families commonly used in several countries for treatment of $M$. bovis, and their MIC values in cattle population are reported in several studies, allowing a comparison with previous data. Doxycycline and tiamulin were selected to assess the susceptibility of $M$. bovis to new antimicrobials, because they are not registered in the European Union for the treatment of dairy cattle. Among the agents of the different antimicrobial classes, the macrolides showed the highest concentration to inhibit $90 \%$ of isolates $\left(\mathrm{MIC}_{90}\right)$, all above the highest concentration tested: $>8$ $\mu \mathrm{g} / \mathrm{mL}$ for erythromycin, $>16 \mu \mathrm{g} / \mathrm{mL}$ for spiramycin, and $>32 \mu \mathrm{g} / \mathrm{mL}$ for tilmicosin and tylosin. Also the $\mathrm{MIC}_{90}$ of lincomycin was above the highest concentration tested $(>32 \mu \mathrm{g} / \mathrm{mL})$, but the distribution of the MIC values was almost perfectly bimodal: 41 isolates had a MIC $\leq 0.5 \mu \mathrm{g} / \mathrm{mL}$ and 30 isolates $>32 \mu \mathrm{g} / \mathrm{mL}$. Oxytetracycline had a 2-fold higher concentration to

Received October 28, 2015.

Accepted April 9, 2016.

${ }^{1}$ Corresponding author: abarberio@izsvenezie.it inhibit $50 \%$ of isolates ( 2 vs. $0.5 \mu \mathrm{g} / \mathrm{mL}$ ) and 1-fold higher $\mathrm{MIC}_{90}$ (4 vs. $2 \mu \mathrm{g} / \mathrm{mL}$ ) than doxycycline. Enrofloxacin and florfenicol had both a $\mathrm{MIC}_{90}$ of $2 \mu \mathrm{g} / \mathrm{mL}$, whereas tiamulin had a $\mathrm{MIC}_{90}$ of $0.5 \mu \mathrm{g} / \mathrm{mL}$. Significant differences on the MIC values were found among the 3 countries for several antimicrobials: compared with Germany, Belgium and Italy showed significantly higher MIC for lincomycin, spiramycin, and tylosin, and lower for oxytetracycline and florfenicol. The Belgian isolates showed the lowest MIC for enrofloxacin compared with Germany and Italy. The MIC results obtained in our study suggest the presence of a high level of resistance of $M$. bovis isolates originating from milk to macrolides in all countries involved in this study. On the contrary, a low level of resistance was found against the antimicrobials that are not used in cattle, such as tiamulin and doxycycline, highlighting a possible link between antimicrobial treatments and development of resistance in the studied M. bovis population.

Key words: Mycoplasma bovis, minimum inhibitory concentration, antimicrobials, dairy cattle

\section{Short Communication}

Mycoplasma bovis is a microorganism lacking a cell wall, belonging to the class of Mollicutes. In cattle it can induce respiratory disease, otitis media, arthritis, mastitis, and keratoconjunctivitis (Maunsell et al., 2011). This bacterium is considered to be one of the major emerging pathogens in cattle herds of industrialized countries threatening livestock production (Nicholas, 2011).

Mycoplasma bovis mastitis can occur with clinical signs, for example milk abnormalities such as flaky 
sediments in a watery or serous fluid, swelling, and udder induration (Bushnell, 1984; Maunsell et al., 2011); however, subclinical cases of mastitis have been described (González and Wilson, 2003; Maunsell et al., 2011). Mycoplasma bovis mastitis has been considered contagious in nature and is transmitted from infected to uninfected udders mostly at milking time. Still, the transmission may less commonly occur from pathways other than those associated with milking (Fox et al., 2003). Some studies have highlighted the ability of $M$. bovis to colonize multiple body sites (Punyapornwithaya et al., 2010), and Fox (2012) postulated that transmission of $M$. bovis associated with bovine IMI may occur within the cow internally, from one infected organ site to the udder or vice versa. Also, between-cow transmission by shedding of the pathogen through external mucosal surfaces of an infected or colonized animal to a naïve animal has been mentioned (Fox, 2012).

To date, no effective vaccine exists to prevent $M$. bovis infection in cattle (Mulongo et al., 2013). Antibiotic treatment is used to control the respiratory and arthritic forms of the disease; however, Mycoplasma mastitis responds poorly to antimicrobial treatment (González and Wilson, 2003; Thomas et al., 2003; Maunsell et al., 2011; Lerner et al., 2014). Increasing antimicrobial resistance of $M$. bovis isolates has been reported in several European countries and in the United States. High levels of resistance were found, especially against tylosin and tilmicosin, both in the United States and in Europe (Rosenbusch et al., 2005; Soehnlen et al., 2011; Ayling et al., 2014; Gautier-Bouchardon et al., 2014; Sulyok et al., 2014). The large majority of the studies on the antimicrobial susceptibility have been performed on isolates obtained from the respiratory tract of beef and dairy cattle, whereas few data are available on mastitis isolates (Soehnlen et al., 2011; Kawai et al., 2014) because antimicrobial treatment of $M$. bovis mastitis is usually unsuccessful (González and Wilson, 2003). The evaluation of the antimicrobial susceptibility profiles of M. bovis isolated from bovine milk samples could be helpful to assess the levels of antimicrobial resistance originating from distinct dairy cattle populations, given that $M$. bovis mastitis, when recognized, is typically not treated with antibiotic therapy or, if the pathogen is undetected, is treated with unsuitable antimicrobials, such as betalactams.

The objective of this study was to assess the in vitro antimicrobial susceptibility of 73 isolates of $M$. bovis identified in milk samples of dairy herds of Belgium, Germany, Italy, and to evaluate the overall patterns of antimicrobial resistance and the differences among the 3 countries. Moreover the aim was to evaluate if the $M$. bovis antimicrobial resistance profiles from dairy cattle were similar or different compared with published studies with beef cattle isolates.

For the assessment of antimicrobial susceptibility macrolides, florfenicol, oxytetracycline, and enrofloxacin were chosen because they represent antimicrobial families commonly used for the treatment of $M$. bovis infection in several countries. The MIC values in cattle isolate populations have been reported in several studies, allowing a comparison with previous data (Rosenbusch et al., 2005; Soehnlen et al., 2011; Ayling et al., 2014; Gautier-Bouchardon et al., 2014; Sulyok et al., 2014). Among these antimicrobials, tilmicosin is used only in beef cattle or dairy youngstock due to the long milk withdrawal time, whereas all the others are used both in dairy and beef cattle. Among antimicrobials currently used for food animals in the European Union, but yet not registered for treatment in cattle, doxycycline and tiamulin were selected to assess the susceptibility of $M$. bovis. Tiamulin was chosen because is reported as very effective against several mycoplasmas including M. bovis ((Thomas et al., 2003; Gigueré, 2013). Doxycycline was selected to compare its antimicrobial activity against $M$. bovis with oxytetracycline, which belongs to the same antibiotic family but has been widely used in cattle from decades.

The isolates (73) used in our study were part of the laboratory culture collection of 4 European laboratories, located in Belgium (Flanders Milk Control Center, Lier), in Germany (University of Applied Science and Arts-Microbiology, Hannover), and in Italy (Istituto Zooprofilattico Sperimentale delle Venezie (IZSVe), Legnaro, and Istituto Zooprofilattico Sperimentale della Lombardia e dell'Emilia, Brescia]. The isolates were selected from 68 individual cows and 4 bulk tank milk samples collected in 4 Belgian, 17 German, and 34 Italian dairy farms located in different areas of the country without epidemiological relation among them.

All the antimicrobial susceptibility tests were performed at the laboratory of IZSVe and all isolates were grown to reach the log phase, stored in PPLO broth medium with the addition of sterile glycerol $(5 \% \mathrm{vol} /$ $\mathrm{vol}$ ), frozen at $-20^{\circ} \mathrm{C}$ at the different labs, and shipped to the IZSVe laboratory for MIC determination. The MIC were determined following the guidelines for $M y$ coplasma MIC testing (Hannan, 2000), and the Clinical and Laboratory Standards Institute (CLSI) guidelines for performing MIC in human Mycoplasma isolates (CLSI, 2011).

All the isolates, after their arrival, were inoculated in $2 \mathrm{~mL}$ of PPLO broth medium with added phenol red (Acumedia-Neogen, Lansing, MI), and incubated at 37 $\pm 1{ }^{\circ} \mathrm{C}$ under $5 \% \mathrm{CO}_{2}$ conditions. Broths were checked daily for changes in color or turbidity; then they were 
inoculated onto PPLO agar medium (Acumedia-Neogen) and checked daily for the presence of $M$. bovis colonies. To confirm the identification of M. bovis, DNA was extracted from broth of positive samples and a 16SrDNA PCR and denaturing gradient gel electrophoresis method were performed as described by McAuliffe et al. (2005).

Each $M$. bovis isolate was submitted to 2 consecutive serial passages both in liquid and solid PPLO medium, according to the guidelines for Mycoplasma MIC testing (Hannan, 2000), and finally propagated in $10 \mathrm{~mL}$ of PPLO broth without inhibitors. The bacterial stock solution was spiked in 10 subaliquots and stored at least $24 \mathrm{~h}$ at $-80^{\circ} \mathrm{C}$. The day after, an aliquot was thawed and used for the evaluation of the titer by the unit changing color (UCC/mL; Hannan, 2000; Blodgett, 2010). When the titer was available, a new subaliquot of approximately $10^{4} \mathrm{UCC} / \mathrm{mL}$ was inoculated in each well of a 96-well commercial ready-for-use plate (Merlin Diagnostika, Micronaut-S, Berlin, Germany) that contained 10 freeze-dried antimicrobials (Table 1).

Plates were incubated aerobically at $37 \pm 1^{\circ} \mathrm{C}$ for at least $18 \mathrm{~h}$ and then checked thrice daily to confirm the growth of the positive control well. Each isolate was tested in duplicate and reference strain ATCC 25523 was tested as quality control. The MIC was determined when the positive control well showed an evident growth. All the isolates grew within $24 \mathrm{~h}$ from beginning of incubation.

The MIC value of each isolate, expressed as micrograms per milliliter, was defined as the lowest concentration of the antimicrobial agent that completely inhibited the growth after the incubation period. When the growth was not inhibited at the highest antimicrobial concentration, the MIC was expressed as greater than the highest concentration; when the growth was inhibited at the lowest concentration, the MIC was expressed as lower or equal to this concentration. For each tested antimicrobial the following parameters were calculated: MIC dilution range used, MIC range, mode, defined as the value that occurs more often in the data set, as well as the lowest concentrations that inhibit 50 and $90 \%$ of the isolates $\left(\mathrm{MIC}_{50}\right.$ and $\mathrm{MIC}_{90}$, respectively). The variability within antibiotic MIC values were measured by the median absolute deviation, which is a statistic dispersion index more robust than standard deviation. Median absolute deviation is defined as the median of the absolute deviation of each data from the data median.

Statistical analysis was performed to evaluate the differences among MIC of the 3 countries using SPSS 22.0 software for windows (IBM, Armonk, NY). The overall variance among the isolates MIC was evaluated using the Kruskal-Wallis test for each antimicrobial, and then post-hoc comparisons among the MIC of the 3 countries were performed using the Wilcoxon rank-sum test. Any $P$-values $<0.05$ were considered statistically significant for the Kruskal-Wallis test, whereas Wilcoxon test was considered significant only with a $P$-value $<0.0167$, corresponding to the value of 0.05 divided by the number of comparisons (3) to be tested (Belgium vs. Germany, Belgium vs. Italy, Germany vs. Italy).

The antimicrobial susceptibility profiles of all the isolates are summarized in Table 1. Among the antimicrobials tested, all macrolides (erythromycin, spiramycin, tilmicosin, and tylosin) showed the highest $\mathrm{MIC}_{90}$ val-

Table 1. Antimicrobial susceptibility patterns of the 73 Mycoplasma bovis isolates identified in milk, with dilution range, $\mathrm{MIC}$ range, mode, the concentration to inhibit $90 \%$ of isolates $\left(\mathrm{MIC}_{90}\right)$, and median absolute deviation (MAD) for each antibiotic tested

\begin{tabular}{|c|c|c|c|c|c|}
\hline $\begin{array}{l}\text { Test } \\
\text { agent }\end{array}$ & $\begin{array}{l}\text { Dilution range } \\
\qquad(\mu \mathrm{g} / \mathrm{mL})\end{array}$ & $\begin{array}{l}\text { MIC range } \\
(\mu \mathrm{g} / \mathrm{mL})\end{array}$ & $\begin{array}{c}\text { Mode } \\
(\mu \mathrm{g} / \mathrm{mL})\end{array}$ & $\begin{array}{c}\mathrm{MIC}_{90} \\
(\mu \mathrm{g} / \mathrm{mL})\end{array}$ & $\begin{array}{c}\text { MAD } \\
(\mu \mathrm{g} / \mathrm{mL})\end{array}$ \\
\hline \multicolumn{6}{|l|}{ Quinolones } \\
\hline Enrofloxacin & $0.125-32$ & $\leq 0.125-16$ & 0.25 & 2 & 0.25 \\
\hline \multicolumn{6}{|l|}{ Lincosamides } \\
\hline Lincomycin & $0.5-32$ & $\leq 0.5->32$ & $\leq 0.5$ & $>32$ & 0.5 \\
\hline \multicolumn{6}{|l|}{ Macrolides } \\
\hline Erythromycin & $0.5-8$ & $>8$ & $>8$ & $>8$ & 8 \\
\hline Spiramycin & $0.5-16$ & $\leq 0.5->16$ & 16 & $>16$ & 8 \\
\hline Tilmicosin & $0.625-32$ & $2->32$ & $>32$ & $>32$ & 32 \\
\hline Tylosin & $0.625-32$ & $0.125->32$ & $>32$ & $>32$ & 32 \\
\hline \multicolumn{6}{|l|}{ Phenicols } \\
\hline Florfenicol & $0.5-16$ & $0.5-4$ & 1 & 2 & 1 \\
\hline \multicolumn{6}{|l|}{ Pleuromutilins } \\
\hline Tiamulin & $0.625-32$ & $0.625-0.5$ & 0.125 & 0.5 & 0.25 \\
\hline \multicolumn{6}{|l|}{ Tetracyclines } \\
\hline Doxycycline & $0.125-32$ & $0.25-32$ & 0.5 & 2 & 0.5 \\
\hline Oxytetracycline & $0.5-32$ & $1->32$ & 4 & 4 & 2 \\
\hline
\end{tabular}


ues, all above the highest concentration tested. This is in agreement with previous report on $M$. bovis isolates originating from milk (Soehnlen et al., 2011; Kawai et al., 2014) and the respiratory tract of beef and dairy cattle (Rosenbusch et al., 2005; Uemura et al., 2010; Soehnlen et al., 2011; Ayling et al., 2014; GautierBouchardon et al., 2014; Sulyok et al., 2014). The macrolide with the lowest $\mathrm{MIC}_{50}(\leq 0.05 \mu \mathrm{g} / \mathrm{mL})$ was spiramycin, with 27 isolates $(37 \%)$ having an MIC $\leq 0,5$ $\mu \mathrm{g} / \mathrm{mL}$, the lowest concentration tested. Among macrolides, it is interesting to note the differences between tylosin and tilmicosin: for tilmicosin all the isolates but one had a MIC above the highest concentration tested, whereas the distribution of MIC values for tylosin was wider, with 43 isolates (59\%) ranging from 0.125 to 32 $\mu \mathrm{g} / \mathrm{mL}$ and 30 isolates $(61 \%)>32 \mu \mathrm{g} / \mathrm{mL}$. A different level of resistance among these antimicrobials has already been reported (Gerchman et al., 2009; Sulyok et al., 2014; Ayling et al., 2014). In a previous study (Lerner et al., 2014) it has been highlighted that, although tylosin and tilmicosin share the same ribosomal binding site, a combination of point mutations in the 23S rRNA genes is necessary to achieve a high level of resistance to tylosin, whereas mutations in only a single domain may alone confer high resistance to tilmicosin. Nevertheless, this difference is peculiar because tilmicosin in the European Union is not used at all in dairy cows due to the long milk withdrawal time, whereas tylosin is frequently used also in dairy cows.

Lincomycin, an antimicrobial belonging to the class of lincosamides, showed a bimodal distribution of the MIC values (Figure 1): 41 isolates (56\%) had a MIC $\leq 0.5 \mu \mathrm{g} / \mathrm{mL}$ and $30(41 \%)$ were $>32 \mu \mathrm{g} / \mathrm{mL}$. Resistance to lincosamides is commonly associated with resistance to macrolides and streptogramins group B, and this is due to isolates harboring erm genes and producing an enzyme that methylates the 23S rRNA (Gigueré, 2013). The different resistance pattern observed in lincomycin, compared with macrolides, could be explained by the presence of a dissociated inducible cross-resistance in which bacteria resistant to macrolides are fully susceptible to lincosamides, but can rapidly develop resistance also to lincosamides when exposed to macrolides (Gigueré, 2013). The MIC values distribution for enrofloxacin (Figure 1) was unimodal and both $\mathrm{MIC}_{50}(0.25 \mu \mathrm{g} / \mathrm{mL})$ and $\mathrm{MIC}_{90}(2 \mu \mathrm{g} / \mathrm{mL})$ were much lower compared with those for the macrolides. These results were in agreement with the data of previous studies performed in Belgium, France, and Japan (Thomas et al., 2003; Gautier-Bouchardon et al., 2014; Kawai et al., 2014), irrespective whether isolates originated from the respiratory tract of beef and dairy cattle or from milk. Two tetracyclines were tested, oxy- tetracycline and doxycycline, the latter not approved in the European Union for treatment in dairy cattle. Oxytetracycline had a 2-fold higher $\mathrm{MIC}_{50}$ (2 vs. 0.5 $\mu \mathrm{g} / \mathrm{mL}$ ) and 1-fold higher $\mathrm{MIC}_{90}(4$ vs. $2 \mu \mathrm{g} / \mathrm{mL}$ ) than doxycycline. The MIC values for oxytetracycline were lower than those reported in other studies (Thomas et al., 2003; Soehnlen et al., 2011; Gautier-Bouchardon et al., 2014; Kawai et al., 2014; Sulyok et al., 2014), in which $\mathrm{MIC}_{50}$ and $\mathrm{MIC}_{90}$ were also 4-fold greater than in our study. For florfenicol, an antimicrobial used for bovine respiratory disease treatment in cattle, the $\mathrm{MIC}_{50}$ $(1 \mu \mathrm{g} / \mathrm{mL})$ and the $\mathrm{MIC}_{90}(2 \mu \mathrm{g} / \mathrm{mL})$ were much lower than those reported in other studies, as well as when the isolates were obtained from milk (Soehnlen et al., 2011; Gautier-Bouchardon et al., 2014). Also tiamulin, an antimicrobial belonging to the class of pleuromutilins and currently approved only for treatment of swine and poultry in the European Union, was tested and deemed very active against $M$. bovis. Both $\mathrm{MIC}_{50}(0.25 \mu \mathrm{g} / \mathrm{mL})$ and $\mathrm{MIC}_{90}(0.5 \mu \mathrm{g} / \mathrm{mL})$ values of tiamulin were very low, in agreement with the values reported in literature (Thomas et al., 2003; Gigueré, 2013).

In Table 2 the value of the $\mathrm{MIC}_{90}$ of the isolates grouped by countries are shown. Significant differences $(P<0.05)$ of the MIC values were found among the 3 countries for the following compounds: enrofloxacin, florfenicol, lincomycin, oxytetracycline, spiramycin, tiamulin, and tylosin. In detail, compared with Germany, Belgium and Italy showed significantly $(P<$ 0.0167 ) higher MIC for lincomycin, spiramycin, and tylosin, and lower for oxytetracycline and florfenicol. The Belgian isolates showed the lowest MIC for enrofloxacin compared with Germany and Italy.

Taking into account the limited number of isolates tested, and the possibility that differences in resistance across countries could be influenced by differences among strains of the microorganism, this evaluation can only provide some considerations about differences among the 3 countries. First, no single country showed a greater level of resistance for all the antimicrobials, yet the differences among the 3 countries were limited to some specific antimicrobials. The different MIC patterns in the 3 countries could reflect different market availability for the different antimicrobials or different treatment procedures for diseases.

Because of the absence of CLSI-approved and -standardized breakpoints for $M$. bovis, the isolates were not classified as susceptible, intermediate or resistant to the different antimicrobials. Nevertheless, the MIC results obtained in our study suggest the presence of a high level of resistance to macrolides in all the countries involved in this study. This is remarkable, especially for tilmicosin and tylosin, as they are considered the first 

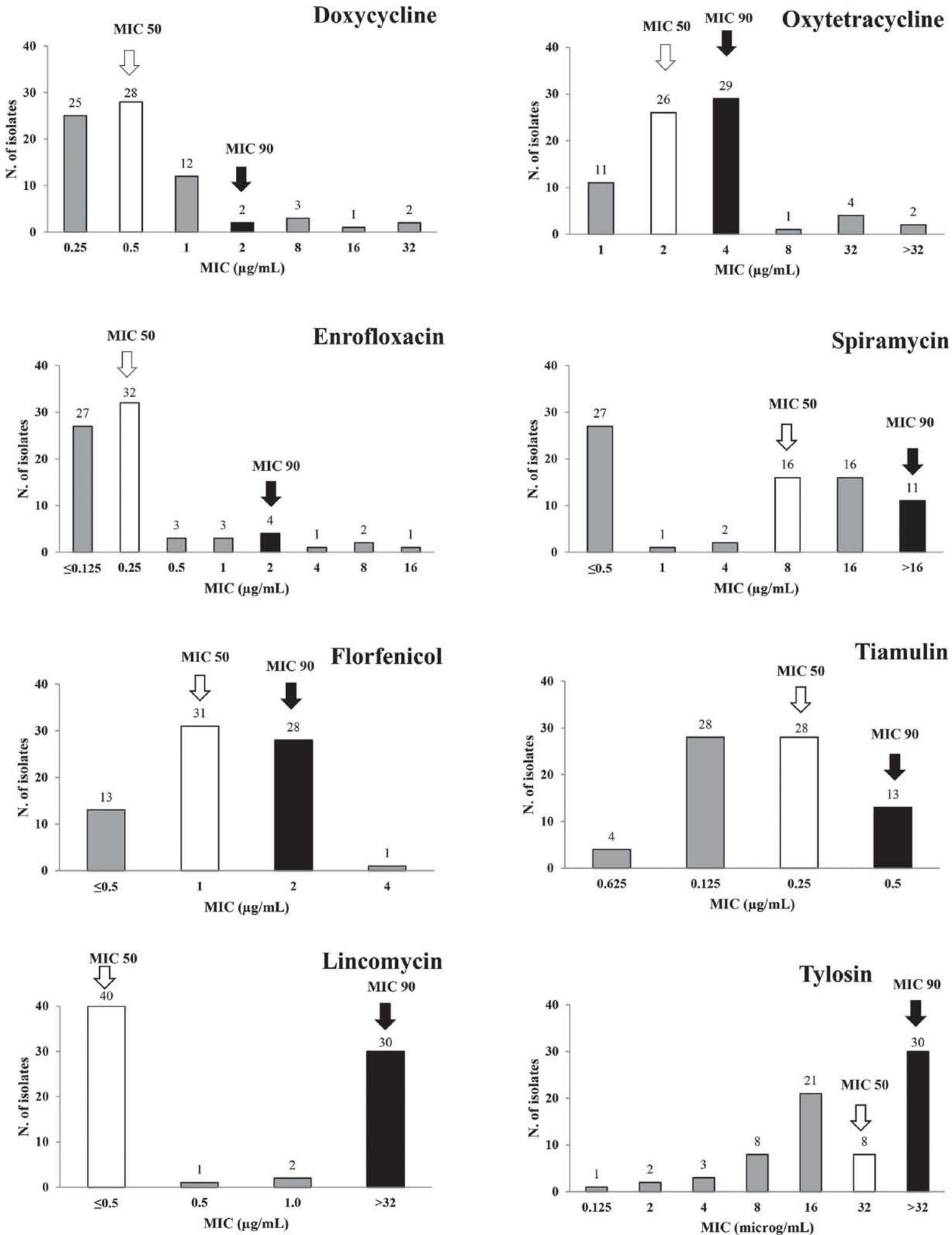

Figure 1. Minimum inhibitory concentration distribution $(\mu \mathrm{g} / \mathrm{mL})$ of the 73 Mycoplasma bovis isolates for the following antimicrobials: doxycycline, enrofloxacin, florfenicol, lincomycin, oxytetracycline, spiramycin, tiamulin, and tylosin. White bars and arrows indicate the concentration to inhibit $50 \%$ of the isolates $\left(\mathrm{MIC}_{50}\right.$ values), black bars and arrows are the concentration to inhibit $90 \%$ of the isolates (MIC 90 values), gray bars indicate the other MIC values. On the top of each bar is the number of isolates for each MIC value. The MIC values that were above the dilution range, when the growth was not inhibited at the highest antimicrobial concentration, are marked as greater than; values below the dilution range, when the growth was inhibited also at the lowest concentration, are marked less than or equal to. Erythromycin and tilmicosin have not been included because their distribution was one-dimensional. 
Table 2. Comparison among the concentrations to inhibit 50 and $90 \%$ of the isolates $\left(\mathrm{MIC}_{50}\right.$ and $\mathrm{MIC}$, respectively) of the antimicrobials tested sorted for countries

\begin{tabular}{|c|c|c|c|c|c|c|c|}
\hline Test agent & \multicolumn{3}{|c|}{$\mathrm{MIC}_{50}(\mu \mathrm{g} / \mathrm{mL})$} & \multicolumn{3}{|c|}{$\mathrm{MIC}_{90}(\mu \mathrm{g} / \mathrm{mL})$} & $\begin{array}{c}\text { Overall variance }^{1} \\
(P \text {-value })\end{array}$ \\
\hline \multicolumn{8}{|l|}{ Lincosamides } \\
\hline Lincomycin & $>32$ & $\leq 0.5$ & 1 & $>32$ & $>32$ & $>32$ & $0.009^{*}$ \\
\hline \multicolumn{8}{|l|}{ Macrolides } \\
\hline Tilmicosin & $>32$ & $>32$ & $>32$ & $>32$ & $>32$ & $>32$ & 1 \\
\hline Tylosin & 32 & 16 & 32 & $>32$ & $>32$ & $>32$ & $0.001^{*}$ \\
\hline \multicolumn{8}{|l|}{ Phenicols } \\
\hline Florfenicol & 1 & 2 & 1 & 2 & 2 & 2 & $0.000^{*}$ \\
\hline \multicolumn{8}{|l|}{ Pleuromutilins } \\
\hline Tiamulin & 0.25 & 0.125 & 0.25 & 0.5 & 0.25 & 0.5 & $0.000^{*}$ \\
\hline \multicolumn{8}{|l|}{ Tetracyclines } \\
\hline
\end{tabular}

${ }^{1}$ The overall variance among the countries, evaluated using Kruskal-Wallis test, is expressed as $P$-value.

*Statistically significant difference $(P<0.05)$ among the 3 countries.

choice for $M$. bovis therapy in respiratory disease outbreaks. Conversely, low levels of resistance were found against all the antimicrobials that are typically not used in cattle, such as tiamulin and doxycycline, highlighting a possible link between antimicrobial treatments and development of resistance in $M$. bovis population. Of course the high level of resistance found in macrolides could be due to nonspecific resistance mechanism; therefore, further studies are needed to evaluate the reasons of this increasing in antimicrobial resistance.

\section{ACKNOWLEDGMENTS}

The authors thank the European Mastitis Panel (http://www.europeanmastitispanel.eu/default.aspx) for the opportunity to meet and share ideas and Frank Welcome from Cornell University (Ithaca, NY) for English text revision

\section{REFERENCES}

Ayling, R. D., R. S. Rosales, G. Barden, and F. L. Gosney. 2014. Changes in antimicrobial susceptibility of Mycoplasma bovis isolates from Great Britain. Vet. Rec. 175:486. http://dx.doi. org/10.1136/vr.102303.

Blodgett, R. 2010. Most probable number from serial dilutions (Appendix 2). In FDA's Bacteriological Analytical. Accessed October 2015. http://www.fda.gov/Food/FoodScienceResearch/ LaboratoryMethods/ucm109656.htm.

Bushnell, R. B. 1984. Mycoplasma mastitis. Vet. Clin. North Am. Large Anim. Pract. 6:301-312.

CLSI (Clinical and Laboratory Standards Institute). 2011. Methods for Antimicrobial Susceptibility Testing for Human Mycoplasma; approved guideline, M-43. Vol. 31, No. 19. CLSI, Wayne, PA.

Fox, L. K. 2012. Mycoplasma mastitis causes, transmission, and control. Vet. Clin. North Am. Food Anim. Pract. 28:225-237.
Fox, L. K., D. D. Hancock, A. Mickelson, and A. Britten. 2003. Bulk tank milk analysis: Factors associated with appearance of $M y c o-$ plasma spp. in milk. J. Vet. Med. B Infect. Dis. Vet. Public Health 50:235-240.

Gautier-Bouchardon, A. V., S. Ferre', D. Le Grand, A. Paoli, E. Gay, and F. Poumarat. 2014. Overall decrease in the susceptibility of Mycoplasma bovis to antimicrobials over the past 30 years in France. PLoS ONE 9:e87672.

Gerchman, I., S. Levisohn, I. Mikula, and I. Lysnyansky. 2009. In vitro antimicrobial susceptibility of Mycoplasma bovis isolated in Israel from local and imported cattle. Vet. Microbiol. 137:268-275.

Gigueré, S. 2013. Lincosamides, pleuromutilins and streptogramins. Pages 199-210 in Antimicrobial Therapy in Veterinary Medicine. 5th ed. S. Gigueré, J. F. Prescott, P. M. Dowling, ed. John Wiley \& Sons Inc. Ames, IA.

González, R. N., and D. J. Wilson. 2003. Mycoplasma mastitis in dairy herds. Vet. Clin. North Am. Food Anim. Pract. 19:199-221.

Hannan, P. C. 2000. Guidelines and recommendations for antimicrobial minimum inhibitory concentration (MIC) testing against veterinary Mycoplasma species. Vet. Res. 31:373-395.

Kawai, K., H. Higuchi, and H. Iwano. 2014. Antimicrobial susceptibilities of Mycoplasma isolated from bovine mastitis in Japan. Anim. Sci. J. 85:96-99.

Lerner, U., E. Amram, R. D. Ayling, I. Mikula, I. Gerchman, S. Harrus, D. Teff, D. Yogev, and I. Lysnyansky. 2014. Acquired resistance to the 16-membered macrolides tylosin and tilmicosin by Mycoplasma bovis. Vet. Microbiol. 168:365-371.

Maunsell, F. P., A. R. Woolums, D. Francoz, R. F. Rosenbusch, D. L. Step, D. J. Wilson, and E. D. Janzen. 2011. Mycoplasma bovis infection in cattle. J. Vet. Intern. Med. 25:772-783.

McAuliffe, L., R. J. Ellis, J. R. Lawes, R. D. Ayling, and R. A. Nicholas. 2005. 16S rDNA PCR and denaturing gradient gel electrophoresis: a single generic test for detecting and differentiating Mycoplasma species. J. Med. Microbiol. 54:731-739.

Mulongo, M., T. Prysliak, and J. Perez-Casal. 2013. Vaccination of feedlot cattle with extracts and membrane fractions from two $M y-$ coplasma bovis isolates results in strong humoral immune responses but does not protect against an experimental challenge. Vaccine 31:1406-1412.

Nicholas, R. A. 2011. Bovine mycoplasmosis: Silent and deadly. Vet. Rec. 168:459-462

Punyapornwithaya, V., L. K. Fox, D. D. Hancock, J. M. Gay, and J. R. Alldredge. 2010. Association between an outbreak strain causing 
Mycoplasma bovis mastitis and its asymptomatic carriage in the herd: A case study from Idaho, USA. Prev. Vet. Med. 93:66-70.

Rosenbusch, R. F., J. M. Kinyon, M. Apley, N. D. Funk, S. Smith, and L. J. Hoffman. 2005. In vitro antimicrobial inhibition profiles of Mycoplasma bovis isolates recovered from various regions of the United States from 2002 to 2003. J. Vet. Diagn. Invest. 17:436-441.

Soehnlen, M. K., M. E. Kunze, K. E. Karunathilake, B. M. Henwood, S. Kariyawasam, D. R. Wolfgang, and D. M. Jayaro. 2011. In vitro antimicrobial inhibition of Mycoplasma bovis isolates submitted to the Pennsylvania Animal Diagnostic Laboratory using flow cytometry and a broth microdilution method. J. Vet. Diagn. Invest. 23:547-551.
Sulyok, K. M., Z. Kreizinger, L. Fekete, V. Hrivnák, T. Magyar, S. Jánosi, N. Schweitzer, I. Turcsányi, L. Makrai, K. Erdélyi, and M. Gyuranecz. 2014. Antibiotic susceptibility profiles of Mycoplasma bovis strains isolated from cattle in Hungary, Central Europe. BMC Vet. Res. 10:256.http://dx.doi.org/10.1186/s12917-014-0256-x.

Thomas, A., C. Nicolas, I. Dizier, J. Mainil, and A. Linden. 2003. Antibiotic susceptibilities of recent isolates of Mycoplasma bovis in Belgium. Vet. Rec. 153:428-431.

Uemura, R., M. Sueyoshi, and H. Nagatomo. 2010. Antimicrobial susceptibilities of four species of Mycoplasma isolated in 2008 and 2009 from cattle in Japan. J. Vet. Med. Sci. 72:1661-1663. 